\title{
Nanoporous plasmonic metamaterials
}

J. Biener, G. W. Nyce, A. M. Hodge, M. M. Biener, S. O. Kucheyev, A. V. Hamza, S. A. Maier

May 29, 2007

Advanced Materials 
This document was prepared as an account of work sponsored by an agency of the United States Government. Neither the United States Government nor the University of California nor any of their employees, makes any warranty, express or implied, or assumes any legal liability or responsibility for the accuracy, completeness, or usefulness of any information, apparatus, product, or process disclosed, or represents that its use would not infringe privately owned rights. Reference herein to any specific commercial product, process, or service by trade name, trademark, manufacturer, or otherwise, does not necessarily constitute or imply its endorsement, recommendation, or favoring by the United States Government or the University of California. The views and opinions of authors expressed herein do not necessarily state or reflect those of the United States Government or the University of California, and shall not be used for advertising or product endorsement purposes. 


\title{
Nanoporous plasmonic metamaterials
}

\author{
J. Biener ${ }^{*}$, G. W. Nyce, A. M. Hodge, M. M. Biener, and A. V. Hamza \\ Nanoscale Synthesis and Characterization Laboratory, Lawrence Livermore National Laboratory, P.O. Box \\ 808, L-367, Livermore, California 94550, USA \\ S. A. Maier \\ Centre for Photonics and Photonic Materials, University of Bath, Bath BA2 7AY, UK \\ *e-mail: biener2@1lnl.gov
}

We review different routes for the generation of nanoporous metallic foams and films exhibiting well-defined pore size and short-range order. Dealloying and templating allows the generation of both two- and threedimensional structures which promise a well defined plasmonic response determined by material constituents and porosity. Viewed in the context of metamaterials, the ease of fabrication of samples covering macroscopic dimensions is highly promising, and suggests more in-depth investigations of the plasmonic and photonic properties of this material system for photonic applications.

\section{Introduction}

Research in plasmonics is driven by the desire to create materials with designed photonic properties taking advantage of electromagnetic resonances and concomitant enhancement of the electromagnetic near field due to localized surface plasmons.[1, 2] In order to arrive at a macroscopic response, in general ordered arrays of plasmonic constituents such as metal nanoparticles are required.[3, 4] Viewed in the concept of metamaterials,[5] the sub-wavelength periodicity of these structures results in an effective permittivity function. While many photonic applications require materials with well-developed long-range order which, for example, can be prepared by colloidal crystal-templating methods,[6] also disordered materials can exhibit useful photonic function with effective permittivities governed by the underlying short-range order.[7] In this regard, the development of nanoporous metallic materials for photonic and plasmonic applications has recently attracted much interest.

One of the main drivers for the development of plasmonic metamaterials by colloidal self-assembly or material growth processes is the desire to improve the sensitivity of Surface enhanced Raman Spectroscopy (SERS) for fundamental molecular science as well as sensor applications. In this context, the localized plasmon modes sustained by the material are responsible for the large field enhancement achieved on nanoporous metal surfaces.[8] Optimizing the plasmonic response of nanoporous metals requires the capability to fine-tune the feature size (specifically the pores size) of the structure. Despite the fact that long-range order is not required for this application, most of the studies have been performed on periodic structures such as inverse opal crystals.[9, 10] 
Here, we will provide a short review of the synthesis of non-periodic nanoporous metallic materials. In contrast to the periodic structures mentioned above, these materials are not limited to thin films, but can easily be prepared in the form of millimeter-sized 3D objects. Although these structures do not exhibit the long-range order of an inverse opal structure, they still have excellent short-range order and can be very uniform over large volumes. The techniques described in the following have been developed or improved at Lawrence Livermore National Laboratory with the ultimate goal to design a new class of three-dimensional nanoporous metals for high energy density laser experiments. This application requires the fabrication of millimeter-sized, defect-free monolithic samples of nanoporous materials with well-defined pore-size distributions (including hierarchical porosities) and adjustable densities down to a few atomic percent. Besides sensor applications, $[7,11]$ such materials have also very interesting catalytic $[12,13]$ and mechanical properties.[14, 15] Specifically, we will address top-down (e.g. dealloying) and bottom-up techniques (e.g. filter casting and templating) as well as combinations thereof.

\section{Dealloying}

Among the top-down approaches dealloying is an extremely simple and flexible method. In metallurgy, dealloying is defined as selective corrosion (removal) of the less noble constituent from an alloy, usually via dissolving this component in a corrosive environment.[16] This process can lead to spontaneous pattern formation, that is, development of a three-dimensional bicontinuous nanoporous structure while maintaining the original shape of the alloy sample. Thus, virtually any desired shape of the nanoporous material can be obtained by using an appropriately shaped alloy sample. A well-studied example is the formation of nanoporous gold (np-Au) via selective removal of Ag from a Au-Ag alloy.[17] In this system, the removal of silver can be achieved by simply submerging the alloy sample in concentrated nitric acid (so-called "free corrosion") or by applying an electrochemical driving force in a less corrosive electrolyte.[18] The process works best in a narrow compositional range around $\mathrm{Ag}_{0.7} \mathrm{Au}_{0.3}$ [19] and generates a material with a characteristic sponge-like open-cell morphology and a uniform feature size on the nm length scale (Fig. 1) The specific surface area of the materials is in the order of a few $\mathrm{m}^{2} / \mathrm{g}$.[20] Pattern formation during dealloying seems to be a consequence of local surface passivation by clustering of $\mathrm{Au}$ adatoms in combination with continuous etching of Ag.[17] Within this simple model, the length scale of the structure should be a function of the diffusion length of clustering vacancies and $\mathrm{Au}$ adatoms which are continuously generated during dealloying. This conclusion is consistent with the observation that the feature size in $\mathrm{np} A u$ can be controlled by the composition of the electrolyte which in turn controls the diffusion length.[21] For plasmonic applications it is important to note that the process can be easily extended to two-dimensional films by using commercially available white-gold leave with a thickness of a few hundred nanometers.[22]

An equally important aspect of the dealloying process is that the feature size in nanoporous gold can be controlled over a wide range from $10 \mathrm{~nm}$ to the micron length scale through a simple annealing procedure. Most notably, this process does not affect the 
relative density or relative geometry of the material (ligament connectivity or ligament, pore, and sample shape).[23, 24] An example of such an annealing experiment is shown in Figure 2. Note the self-similarity of the structure while increasing the feature size by more than a factor of 30. In a previous SERS study we demonstrated that this effect can be used to fine-tune the plasmonic properties of np-Au.[11] Specifically, we were able to achieve SERS enhancement factors in the range of $10^{9}-10^{11}$ by tuning the feature size of $\mathrm{np} \mathrm{Au}$ to $\sim 500 \mathrm{~nm}$ by annealing at $500{ }^{\circ} \mathrm{C}$. Our results also indicated that the enhancement correlates with the pore size rather than the ligaments, consistent with findings from inverse opal structures.

The dealloying technique can also be used to introduce more complicated morphologies such as hierarchical porosities by using ternary alloys such as $\mathrm{Cu}-\mathrm{Ag}-\mathrm{Au}$ as starting material. The idea is the following: First, the least noble metal is removed thereby creating a nanoporous binary alloy sample. In a second step the material is annealed to the desired feature size. Finally, nano-scale porosity is reintroduced into the ligaments of the structure by removing the second component of the original ternary alloy system (in this example Ag). We have successfully used this technique to prepare low-density $(\sim 10$ at.\% relative density) nanoporous Au samples with a bimodal pore size distribution. The main challenge is the preparation of a homogeneous single phase starting alloy which severely limits the applicability of this approach.

\section{Templating}

Besides dealloying, templating is another approach to incorporate and control porosity.[6, 25-28] Here sacrificial inorganic or organic materials are used as templates to generate a nanostructured porous solid. In particular, the combination of templating and dealloying techniques provides a powerful approach to create materials with complex hierarchical porosities.[29] In the following, some typical examples of the fabrication of nanoporous gold foams based on templating techniques will be described (Scheme 1). The synthesis always starts with the preparation of Au or Ag-Au coated core-shell particles. After assembling (casting) these into a monolithic porous structure, a pure metal foam can be obtained by removing the core material (template). In the case of Ag$\mathrm{Au}$ foams a dealloying step can be added to create hierarchical porosities.

Hollow Ag/Au shells can be fabricated by using silica [30] or polystyrene [31-33] spheres as templates. For our application, polystyrene (PS) is clearly the preferred choice as micro beads with a narrow size distribution are readily available. PS templates can also be easily removed at elevated temperatures or by dissolution in an appropriate organic solvent. Furthermore, PS beads can be metal coated by employing simple electroless plating methods.[32] For the application described here one would typically use PS beads with a diameter ranging from a few hundred nanometers to several microns. The challenge is to prepare the large quantities of metal-coated spheres needed for the fabrication of millimeter-sized foam samples. This makes it necessary to work with highly concentrated solutions of PS beads which are prone to particle aggregation [34] which in turn negatively affects the subsequent casting process. However, highly concentrated colloidal suspensions of PS beads can be stabilized by adding a polymer 
stabilizer such as polyvinylpyrrolidinone (PVP) to the plating bath. Using this approach we were able to coat large quantities of PS beads with Au films of homogeneous thickness and little apparent particle aggregation. Finally, the metal-coated PS beads are isolated and redispersed in distilled water.

The next process step is casting to obtain a monolithic nanoporous sample. The procedure is analogous to slip-casting of ceramic [35] and metal [36] particles in which a suspension of particles is poured into a tube inserted into plaster of paris (Scheme 2). The plaster of paris facilitates the settling of particles by slowly removing the water from the suspension. This simple technique allows one to prepare millimeter-sized monolithic samples of nanoporous materials which are very homogeneous and free of larger defects $(>10 \mu \mathrm{m})$ such as voids. As in the case of dealloying, virtually any desired sample shape can be generated by using an appropriately shaped plaster of paris cast.

The resulting nanoporous monolith consists of randomly packed metal-coated PS spheres with void spaces between individual particles. Figure 3 shows an example of such a structure obtained by filter-casting of gold-coated PS spheres with a diameter of $9.6 \mu \mathrm{m}$. The apparent lack of long-range order can be attributed to the relative polydispersity of the particles, high suspension concentration ( $\approx 10 \mathrm{wt} \%$ ), and high rate of sedimentation. In context of plasmonic applications, it is important to note that the electroless plated gold films on PS beads consist of discrete Au nanoparticles thus adding another length scale to the structure (Figure $3 \mathrm{~b}$ ). This may have important implications as the observation of electromagentic "hot spots" in SERS experiments has been linked to junctions between nanoparticles.[34] Finally, the pure Au foam sample can be obtained by removing the PS template by a simple heat treatment in an inert atmosphere at $400{ }^{\circ} \mathrm{C}$.

Hierachical pore structures can be realized by starting with Ag-Au coated PS core-shell particles and adding an additional dealloying step. Such core-shell structures can be prepared by electroless deposition of silver on Au-coated PS spheres (Scheme 1). The sequence of metal deposition is important since depositing gold on silver may result in competing $\mathrm{Ag}^{\mathrm{O}} / \mathrm{Au}^{3+}$ galvanic and electroless deposition reduction reactions.[37, 38] The Ag-to-Au ratio of the coatings can be adjusted by varying the mole ratios during plating, and typical compositions range from $\mathrm{Ag}_{0.85} \mathrm{Au}_{0.15}$ to $\mathrm{Ag}_{0.7} \mathrm{Au}_{0.3 \cdot[39]}$

Monolithic Ag-Au alloy foam samples can then be obtained by the slip-casting / annealing sequence described above. Here, the heat treatment also leads to the formation of a Ag-Au alloy. In case of the the Ag/Au system discused here, alloying is facilitated by the matching crystalline structure of $\mathrm{Ag}$ and $\mathrm{Au}$ (both fcc with nearly identical lattice constants) and high diffusion rates of silver and gold at elevated temperatures. An example of such a structure made from $10 \mu \mathrm{m} \mathrm{Ag} \mathrm{Ag}_{0.8} \mathrm{Au}_{0.15}$ shells is shown in Figure 4a. The diameter of the shells roughly corresponds to the size of the original PS template, indicating negligable shrinkage upon template removal and good control over the feature size.

Finally, hierarchical pore structures can be generated by dealloying of $\mathrm{Ag}-\mathrm{Au}$ foam samples. Here, the concentration of the nitric acid needs to be carefully adjusted to prevent cracking of the monolithic sample. Due to the very low density of such a material 
( $1.5 \%$ relative density or a void space of $98.5 \%$ ), drying is challenging, particlularly when one intends to make millimeter-sized samples of crack-free material. The samples need to be washed several times in distilled water to remove residual nitric acid and then placed in acetone. Once the water is completely exchanged with acetone, the monoliths can be dried by supercritical $\mathrm{CO}_{2}$ extraction.

An SEM image of a dealloyed shell with a wall thickness of $\sim 200 \mathrm{~nm}$ is shown in Figure $4 \mathrm{~b}$. The shell diameter $(\sim 9.6 \mu \mathrm{m})$ is similar to the diameter of the original template thus suggesting little volume change during dealloying and consequent drying. Thus the process outlined above provides good control over the size of the cavities. The feature size introduced by dealloying is in the range of $10-100 \mathrm{~nm}$, giving rise to a relatively large surface area of $\sim 1.5 \mathrm{~m}^{2} / \mathrm{g}$ which is comparable to that of $\mathrm{np} A u$. Thus this material combines the high surface area of np Au with the morphology control characteristic for templating techniques.

The size of the cavities only depends on the size of the orginal PS template, and consequently much smaller structures than the examples described above can be prepared. Figure 5a shows a porous Ag-Au alloy monolith obtained by slip casting $1 \mu \mathrm{m}$ $\mathrm{Ag}_{70} \mathrm{Au}_{30}$ alloy shells prepared from $1 \mu \mathrm{m}$ PS beads. The Ag-Au alloy shell is approximately $50-100 \mathrm{~nm}$ thick and consists of individual particles rather than a continuous film. As described above, consequent dealloying leads to the formation of a nanoporous $\mathrm{Au}$ foam sample with $1 \mu \mathrm{m}$ cavities and a dealloying-induced porosity with a feature size of $50-100 \mathrm{~nm}$ (Figure 5b).

\section{Summary}

We have reviewed different schemes for the generation of porous metals with welldefined pore-size distributions (including complex hierarchical porosities) and adjustable densities down to a few percent of the full density. The methods described here do not result in the formation of long-range ordered structures, but are capable of delivering homogeneous materials on a millimeter length scale. Furthermore, they provide excellent control over feature size ranging from a few nanometers up to a few micrometers which can be exploited to tune material properties for photonic and plasmonic applications as recently demonstrated. Finally, the technology is not only very flexible in terms of engineering new pore morphologies, but can be easily extended to generate new functional structures, for example, via using active optical materials as templates or by adding these materials during the slip-casting process.

\section{.Acknowledgements}

This work was performed under the auspices of the U.S. Department of Energy by University of California, Lawrence Livermore National Laboratory under Contract W7405-Eng-48. 


\section{References}

[1] W. L. Barnes, A. Dereux, T. W. Ebbesen, Nature 2003, 424, 824.

[2] S. A. Maier, Plasmonics: Fundamentals and Applications (Springer, 2007).

[3] S. A. Maier, H. A. Atwater, Journal of Applied Physics 2005, 98, 011101.

[4] N. Felidj, S. L. Truong, J. Aubard, G. Levi, J. R. Krenn, A. Hohenau, A. Leitner, F. R. Aussenegg, J. Chem. Phys. 2004, 120, 7141.

[5] We use the term "metamaterials" in a broad sense to describe the fact that the properties of the materials described here are strongly affected by their cellular architecture. In this broad sense, long-range order is not a necessary requirement.

[6] O. D. Velev, P. M. Tessier, A. M. Lenhoff, E. W. Kaler, Nature 1999, 401, 548.

[7] F. Yu, S. Ahl, A. M. Caminade, J. P. Majoral, W. Knoll, J. Erlebacher, Anal. Chem. 2006, 78, 7346.

[8] T. A. Kelf, Y. Sugawara, J. J. Baumberg, M. Abdelsalam, P. N. Bartlett, Phys. Rev. Lett. 2005, 95.

[9] P. M. Tessier, O. D. Velev, A. T. Kalambur, J. F. Rabolt, A. M. Lenhoff, E. W. Kaler, J. Am. Chem. Soc. 2000, 122, 9554.

[10] P. Tessier, O. D. Velev, A. T. Kalambur, A. M. Lenhoff, J. F. Rabolt, E. W. Kaler, Adv. Mater. 2001, 13, 396.

[11] S. O. Kucheyev, J. R. Hayes, J. Biener, T. Huser, C. E. Talley, A. V. Hamza, Appl. Phys. Lett. 2006, 89, 053102.

[12] V. Zielasek, B. Jurgens, C. Schulz, J. Biener, M. M. Biener, A. V. Hamza, M. Baumer, Angew. Chem., Int. Ed. 2006, 45, 8241.

[13] C. X. Xu, J. X. Su, X. H. Xu, P. P. Liu, H. J. Zhao, F. Tian, Y. Ding, J. Am. Chem. Soc. 2007, 129, 42.

[14] J. Biener, A. M. Hodge, J. R. Hayes, C. A. Volkert, L. A. Zepeda-Ruiz, A. V. Hamza, F. F. Abraham, Nano Lett. 2006, 6, 2379. 
[15] D. Kramer, R. N. Viswanath, J. Weissmueller, Nano Lett. 2004, 4, 793.

[16] R. C. Newman, S. G. Corcoran, J. Erlebacher, M. J. Aziz, K. Sieradzki, MRS Bulletin 1999, 24, 24.

[17] J. Erlebacher, M. J. Aziz, A. Karma, N. Dimitrov, K. Sieradzki, Nature 2001, 410, 450 .

[18] A. M. Hodge, J. R. Hayes, J. A. Caro, J. Biener, A. V. Hamza, Advanced Engineering Materials 2006, 8, 853.

[19] Au-richer alloys develop a self-passivating Au-rich surface layer, whereas Agricher alloys tend to disintegrate into a powder during dealloying.

[20] D. J. Tulimieri, J. Yoon, M. H. W. Chan, Phys. Rev. Lett. 1999, 82, 121.

[21] A. Dursun, D. V. Pugh, S. G. Corcoran, J. Electrochem. Soc. 2003, 150, B355.

[22] Y. Ding, Y. J. Kim, J. Erlebacher, Adv. Mater. 2004, 16, 1897.

[23] A. M. Hodge, J. Biener, J. R. Hayes, P. M. Bythrow, C. A. Volkert, A. V. Hamza, Acta Mater. 2007, 55, 1343.

[24] R. Li, K. Sieradzki, Phys. Rev. Lett. 1992, 68, 1168.

[25] K. M. Kulinowski, P. Jiang, H. Vaswani, V. L. Colvin, Adv. Mater. 2000, 12, 833.

[26] D. Walsh, L. Arcelli, T. Ikoma, J. Tanaka, S. Mann, Nature Mater. 2003, 2, 386.

[27] G. J. D. Soler-illia, C. Sanchez, B. Lebeau, J. Patarin, Chemical Reviews 2002, 102, 4093.

[28] Y. N. Xia, B. Gates, Y. D. Yin, Y. Lu, Adv. Mater. 2000, 12, 693.

[29] G. W. Nyce, J. R. Hayes, A. V. Hamza, J. H. Satcher, Chem. Mater. 2007, 19, 344.

[30] T. Pham, J. B. Jackson, N. J. Halas, T. R. Lee, Langmuir 2002, 18, 4915.

[31] Z. J. Liang, A. Susha, F. Caruso, Chem. Mater. 2003, 15, 3176. 
[32] W. L. Shi, Y. Sahoo, M. T. Swihart, P. N. Prasad, Langmuir 2005, 21, 1610.

[33] T. H. Ji, V. G. Lirtsman, Y. Avny, D. Davidov, Adv. Mater. 2001, 13, 1253.

[34] A. M. Michaels, J. Jiang, L. Brus, J. Phys. Chem. B 2000, 104, 11965.

[35] W. D. Callister, Materials Science and Engineering: An Introduction, 6th ed. (John Wiley \& Sons, Inc., New York, 2003).

[36] J. R. Hayes, G. W. Nyce, J. D. Kuntz, J. H. Satcher, A. V. Hamza, Nanotechnology in press.

[37] Y. G. Sun, Y. N. Xia, J. Am. Chem. Soc. 2004, 126, 3892.

[38] Y. G. Sun, B. T. Mayers, Y. N. Xia, Nano Lett. 2002, 2, 481.

[39] T. Shibata, B. A. Bunker, Z. Y. Zhang, D. Meisel, C. F. Vardeman, J. D. Gezelter, J. Am. Chem. Soc. 2002, 124, 11989. 
Figure 1. Plan-view (a) and cross-section (b) SEM micrographs showing the characteristic sponge-like open-cell foam morphology of np-Au. The material is very homogeneous and exhibits nanometer-sized pores and ligaments, the latter with a lengthto-diameter aspect ratio close to one. The scale bars are $300 \mathrm{~nm}$.
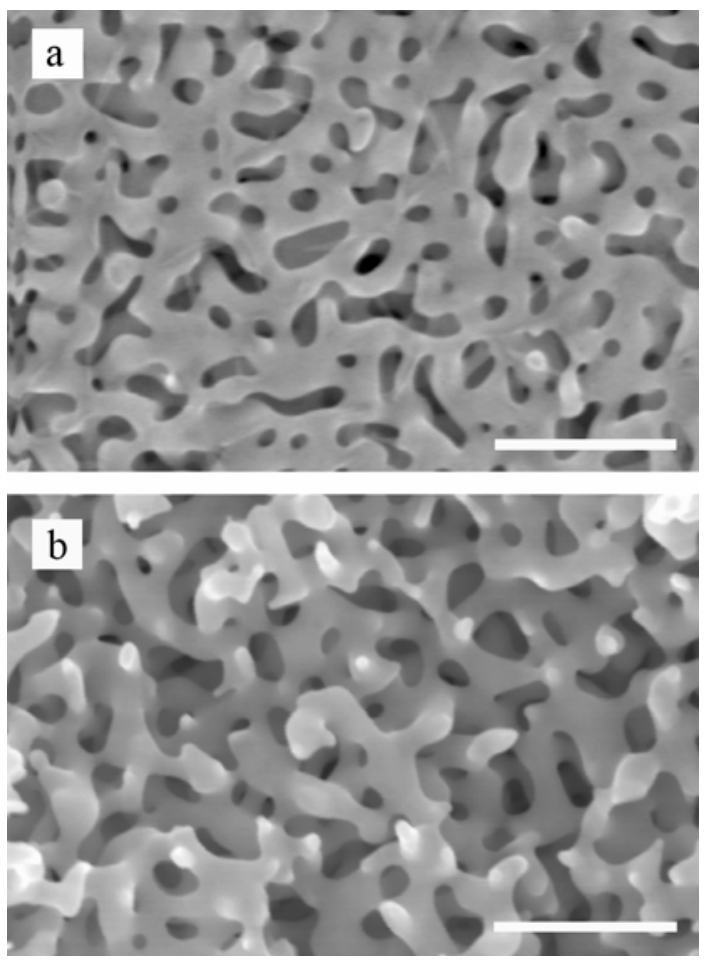
Figure 2. Cross-sectional SEM micrographs of as-prepared (a) and annealed (c-d) np Au. Note the self-similarity of the structure while increasing the feature size by more than a factor of 30 . The relative density of the materials remains constant at approximately $30 \%$.
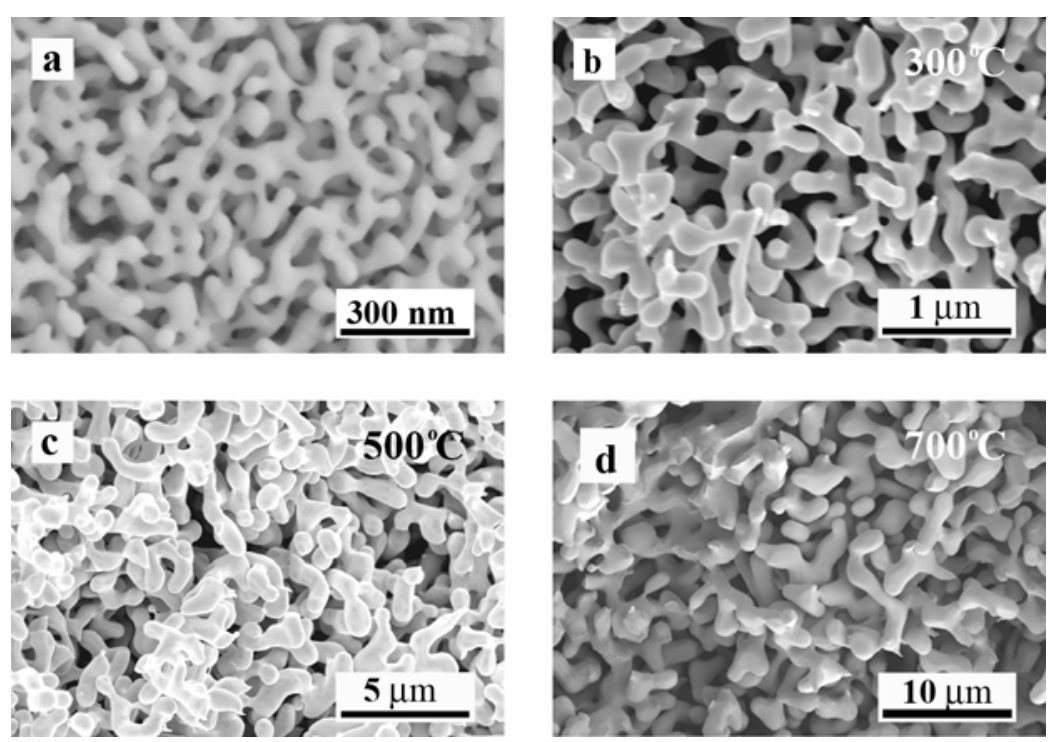
Figure 3. A) SEM micrograph of filter-cast gold coated PS spheres (9.6 $\mu \mathrm{m}$ diameter) fracture surface. The darker interior is the PS core which is exposed due to surface fracture while the lighter exterior surface is the gold surface coating. Removal of the PS template generates a pure $\mathrm{Au}$ foam with a density of $1.7 \mathrm{~g} / \mathrm{cc}(\sim 9 \%$ of the full density of $\mathrm{Au}$ ). B) Higher magnification SEM micrograph of the gold coating. Note that the gold coating is comprised of discrete gold particles, $100-150 \mathrm{~nm}$ in size. The PVP stabilizer likely favors growth of individual gold particles while inhibiting gold film formation.

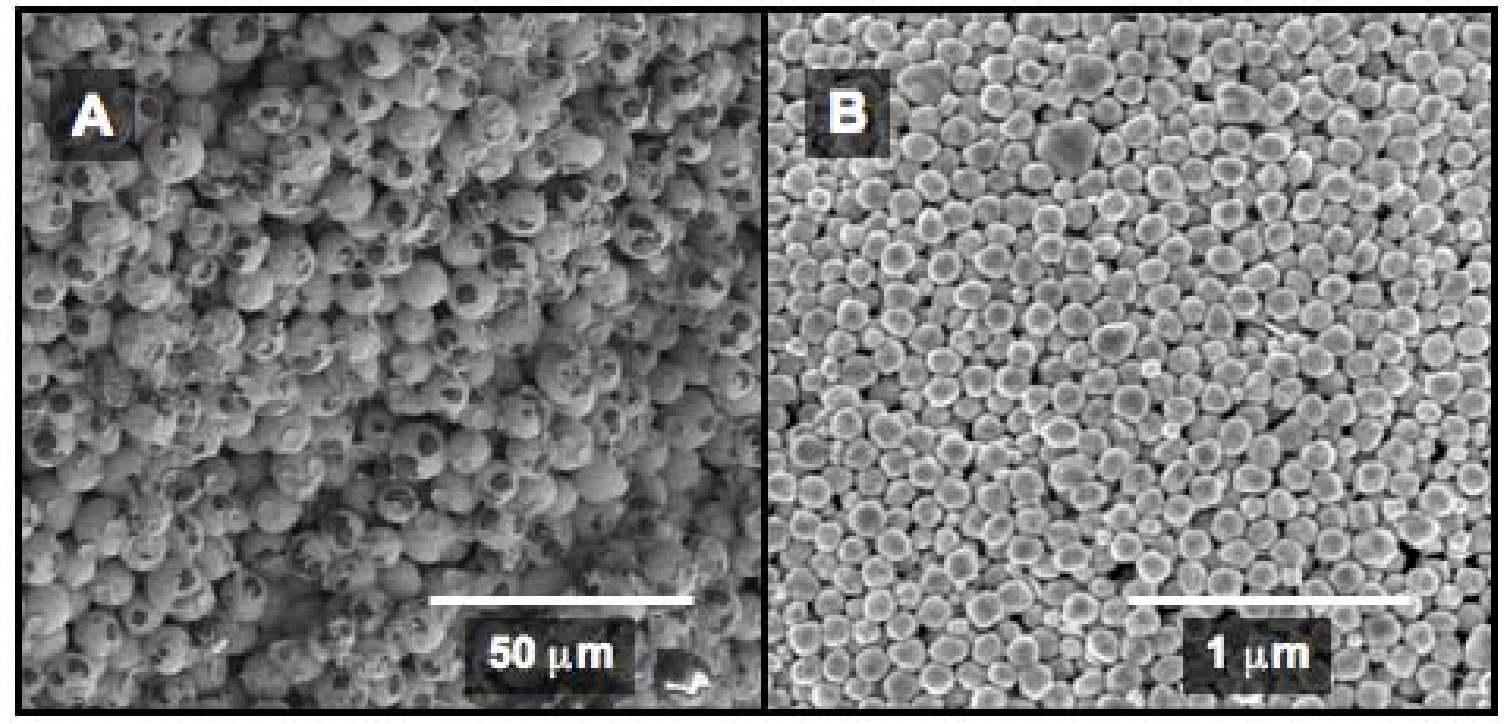


Figure 4. A) Fracture surface of a monolith containing hollow $\mathrm{Ag}_{0.85} \mathrm{Au}_{0.15}$ shells with a density of $0.8 \mathrm{~g} / \mathrm{cc}$ before dealloying. The holes in the shell walls are presumably due to the release of organic volatiles during bakeout. B) Nanoporous hollow gold shell after dealloying. The density of the corresponding Au foam is $0.28 \mathrm{~g} / \mathrm{cc}(1.5 \%$ of the full density of $\mathrm{Au}$ ).

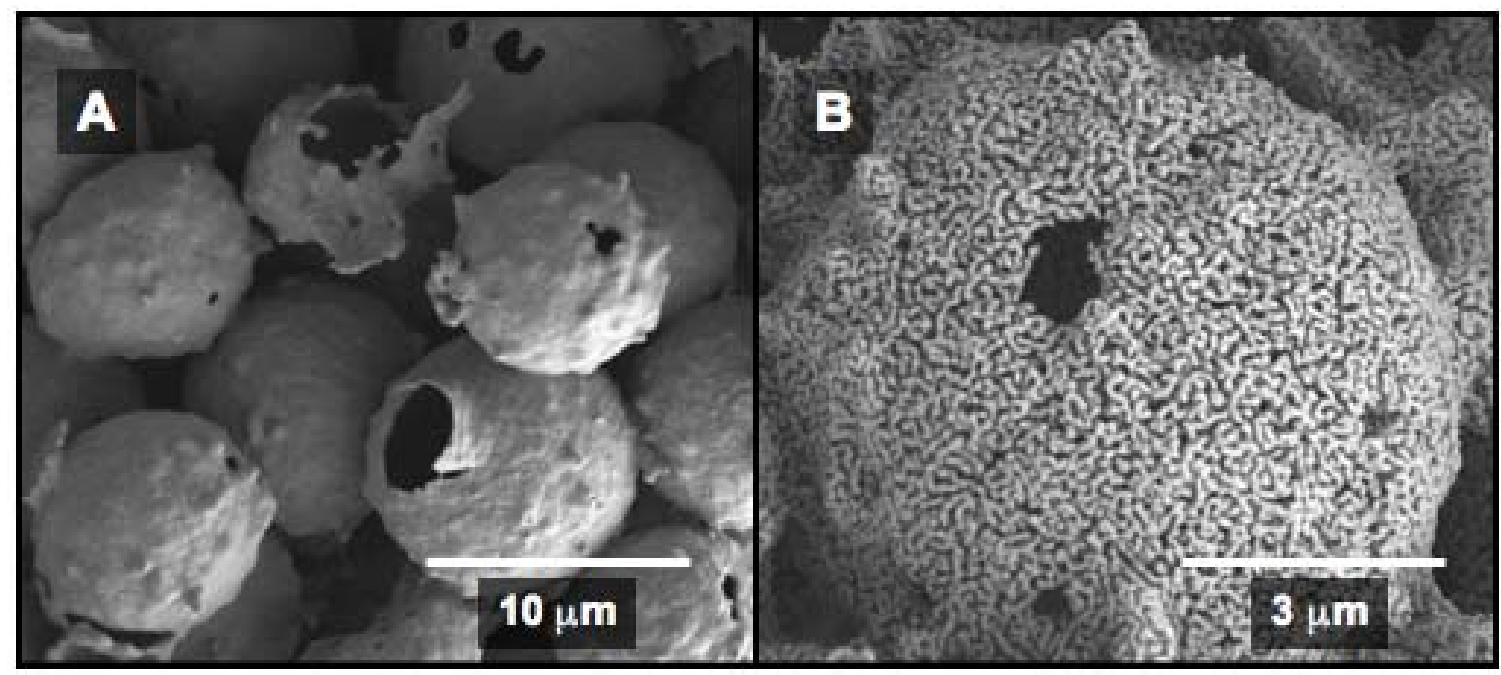


Figure 5. A) Fracture surface of a monolith containing hollow $1 \mu \mathrm{m} \mathrm{Ag}_{0.7} \mathrm{Au}_{0.3}$ shells before dealloying (density $1 \mathrm{~g} / \mathrm{cc}$ ). B) Fracture surface of a monolith containing hollow 1 $\mu \mathrm{m}$ nanoporous Au shells after dealloying (density $0.45 \mathrm{~g} / \mathrm{cc}$ or $2.3 \%$ of the full density of $\mathrm{Au})$.

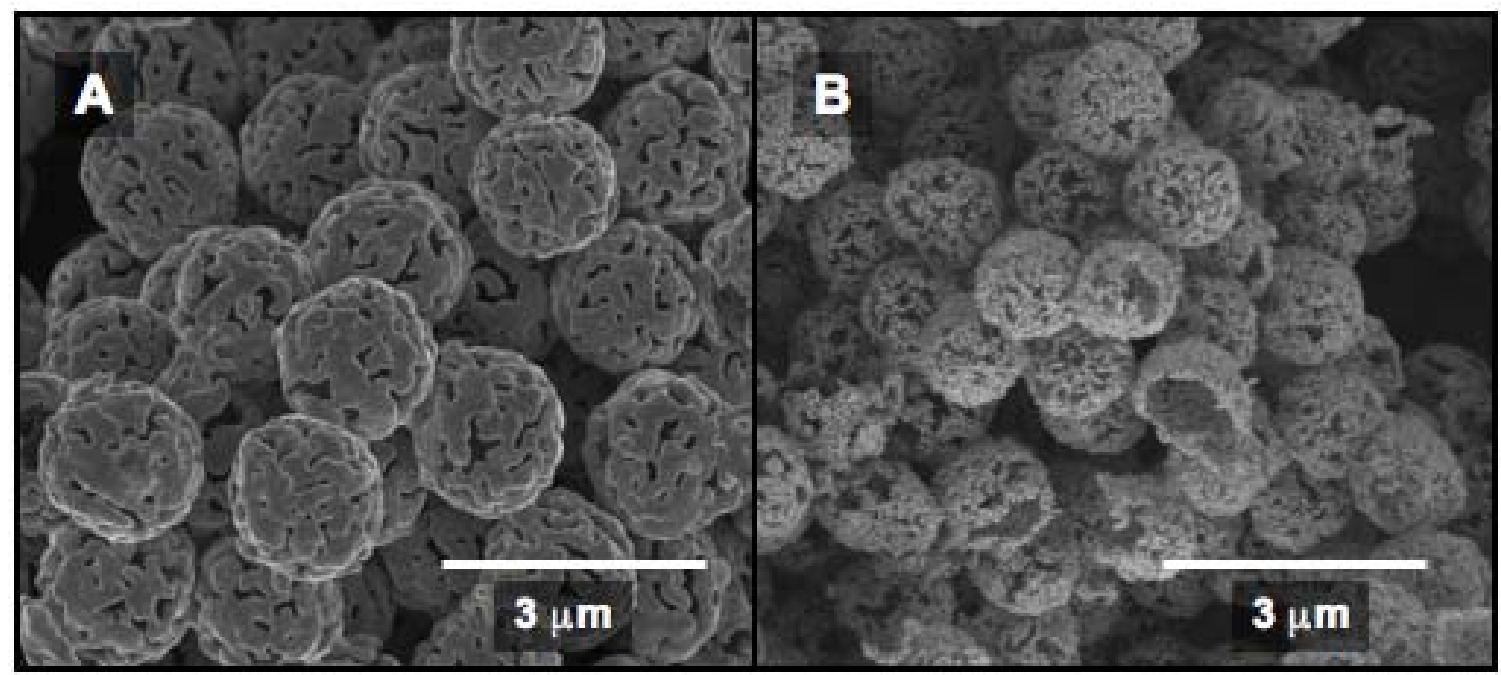



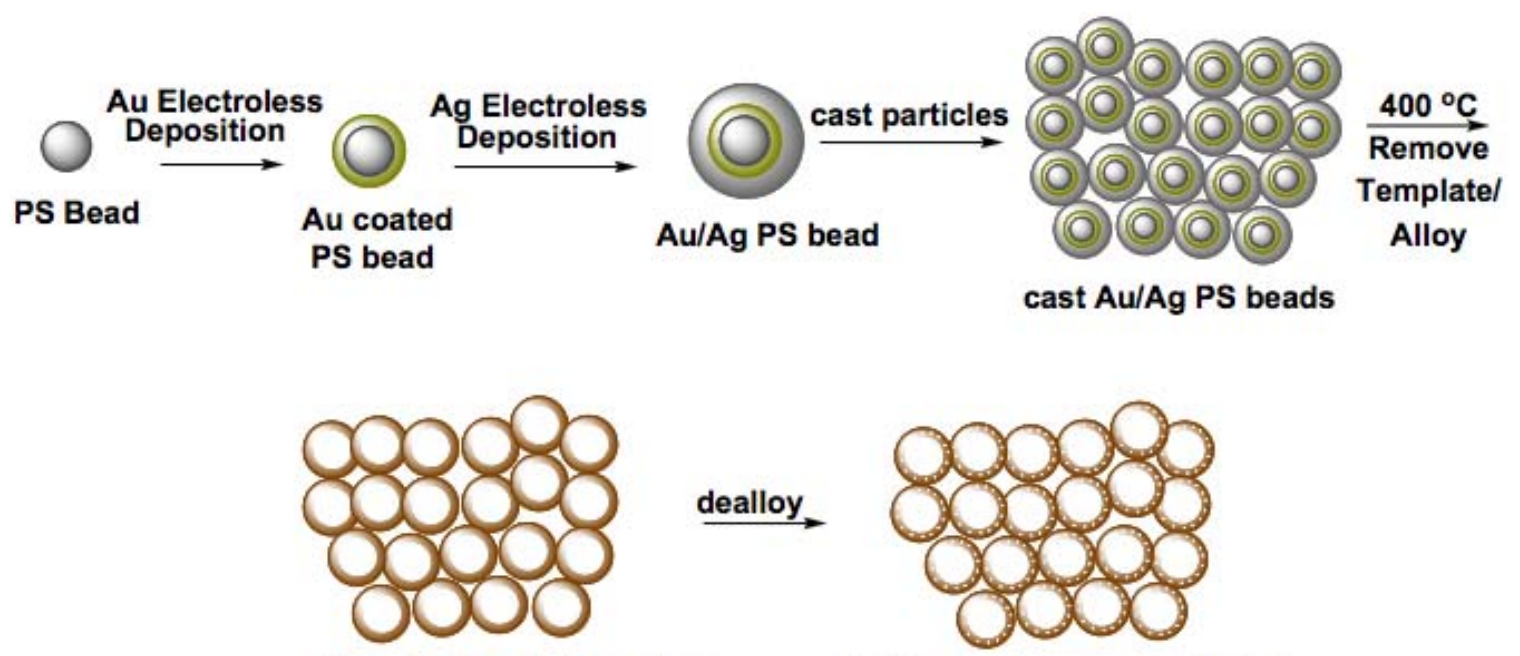

alloyed Au/Ag hollow shells

hollow nanoporous gold shells

Scheme 1. Synthesis of low density gold monoliths comprised of nanoporous hollow gold shells 
Scheme 2. Method for preparing monoliths by casting metal particle suspensions

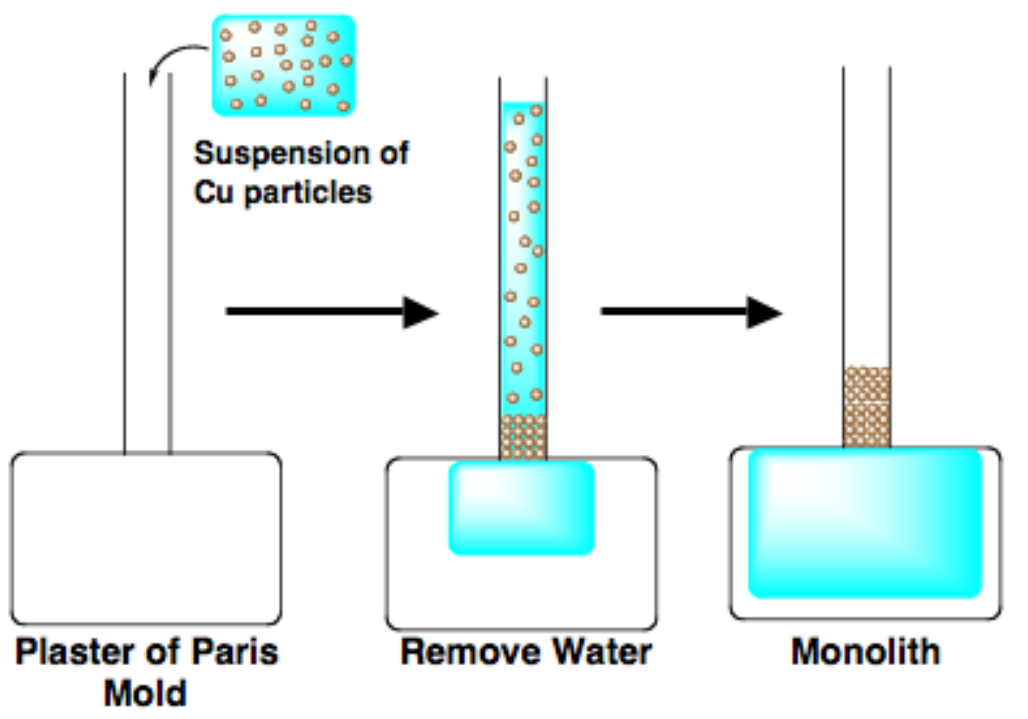

